\title{
A Comparative Evaluation of Simultaneous Bone Marrow Aspiration And Bone Marrow Biopsy Interpretations In Routine Hematology Practice with Special Reference to Flow Cytometry And Cytogenetic Analysis :
}

\author{
*Raka Hota, ${ }^{2}$ Tapaswini Bhuyan ${ }^{3}$ Sukumar Chakrabarty, \\ ${ }^{4}$ R.C Mohanty, ${ }^{5}$ Raghumani Mohanty \\ Dept. Of Pathology, Hi-Tech Medical College And Hospital, Bhubaneswar, Odisha, India \\ Corresponding author: *Raka Hota
}

\begin{abstract}
Introduction: Bone Marrow examination is an important diagnostic tool to evaluate various hematological and non-hematological diseases including both neoplastic and non-neoplastic conditions. The two separate but inter-related techniques are used such as Bone marrow aspiration(BMA) and Bone marrow biopsy(BMB) along with in the recent days introduction of Flow cytometry analysis and cytogenetic study have taken an important diagnostic tool to reach a definitive diagnosis and all these diagnostic processes are complimentary to each other.

Aim and objective: This study is aimed to assess and correlate the diagnostic value of simultaneous BMA and $B M B$ along with Flow cytometric interpretations in different hematological malignancies and non-neoplastic hematological and nonhematological diseases to establish a final diagnosis.

Materials And Methods: This study involved bone marrow analysis in 164 patients out of which in 158 cases both BMA and BMB was performed simultaneously along with flow cytometry and cytogenetic study was done on some hematological malignancies and a correlation was done between all these procedures. Criteria of inclusion included the main indications for performing this procedure, the availability of full clinical data and patient consent.

Result: In the present study, out of 158 cases with simultaneous BMA and BMB procedures were performed simultaneously showed $85.4 \%$ positive correlation between these two procedures. However, it was found that in cases of myelofibrosis, non-hodgkin's lymphoma, myeloproliferative disorders and granulomas, involvement of marrow was detected better in bone marrow biopsies. And also simultaneous flowcytometry and cytogenetic analysis gives strong confirmatory diagnosis in hematological malignancies to know the origin, genetic alteration and minimal residual disease in follow -up cases for targeted therapy.

Conclusion: This study concludes that in routine hematological practice BMA, BMB, Flow cytometry and Cytogenetic, all are complimentary to each other which should be done simultaneously for the better diagnostic and prognostic point of view.
\end{abstract}

Keywords: Bone marrow aspiration, Bone marrow biopsy, Flow cytometry, Cytogenetic

\section{Introduction}

Bone marrow is involved in variety of hematological and non-hematological disorders. The hematological disorders include chronic anemia, pancytopenia, PUO, aplastic anemia, thrombocytopenic purpura, hypersplenism, acute leukemia, myeloproliferative neoplasm (MPN), hemato-lymphoid neoplasm etc. On the other hand non-hematological disorders include infectious diseases infiltrating the bone marrow such as tuberculosis, parasitic infections and metastatic deposits [1]. Although, diseases of bone marrow present with various clinical symptoms and also involve the blood but peripheral blood picture alone does not reflect the nature of disease process. Depending upon diagnosis suspected from the clinical features and peripheral blood examination, indications for bone marrow examination can be summarized. Therefore, complete hematological evaluation of cases where bone marrow examination was indicated includes BMA smear and bone marrow trephine biopsy as they are complementary to each other [2]. In this recent days introduction of Flow cytometry and cytogenetic analysis have taken an important role in routine hematological practice to reach a definitive diagnosis and evaluate the prognostic index and it is helpful in starting a targeted therapy in different hematological malignancies. Hence, we attempted to correlate both these parameters to arrive at a more conclusive final diagnosis. 


\section{Materials And Methods}

This retrospective bone marrow study was performed on total 164 patients from the period of 2015 to 2017 in the dept. of pathology in Hi-Tech Medical College and Hospital, Bhubaneswar, out of which, in 158 cases, both BMA and BMB had been done simultaneously along with detailed clinical history. Most of the patients were presented as fever, anemia, PUO, lymphadenopathy and organomegaly.Other patients, diagnosed as having hematological malignancies on routine peripheral smear examination, were advised for BM study to confirm the diagnosis. The patients had a male to female sex ratio of 3.2:1 and a wide range of age from 15 months to 85 years. [Table 1].It included both indoor and outdoor patients, who were suspected of having bone marrow involvement by any hematological or nonhematological disorders. The relevant clinical history and biodata of the patients was recorded and consent was taken before the procedure and in all aseptic condition under local anesthesia, Bone marrow aspiration and biopsy was done.

Table-1: Age /sex distribution

\begin{tabular}{|ll|ll|ll|ll|}
\hline$\bullet$ & Age group(years) & $\bullet$ & No. of cases & $\bullet$ & Male & $\bullet$ & Female \\
\hline$\bullet$ & $0-10$ & $\bullet$ & 8 & $\bullet$ & 5 & $\bullet$ & 3 \\
\hline$\bullet$ & $11-30$ & $\bullet$ & 40 & $\bullet$ & 32 & $\bullet$ & 8 \\
\hline$\bullet$ & $31-50$ & $\bullet$ & 51 & $\bullet$ & 39 & $\bullet$ & 12 \\
\hline$\bullet$ & $51-70$ & $\bullet$ & 55 & $\bullet$ & 40 & $\bullet$ & 15 \\
\hline$\bullet$ & $>70$ & $\bullet$ & 10 & $\bullet$ & 9 & $\bullet$ & 1 \\
\hline$\bullet$ & total & $\bullet$ & 164 & $\bullet$ & 125 & $\bullet$ & 39 \\
\hline
\end{tabular}

BMA was performed by Salah's marrow puncture needle; smears prepared were stained with Leishman stain. Prussian blue for iron stain was done in selected cases and grading of iron was done. BMB was done by Jamshidi biopsy needle and marrow specimen was fixed in $10 \%$ formalin fixative, decalcified in $10 \%$ formic acid-5\% formaldehyde and processed with paraffin -wax embedding. $1 \mu \mathrm{m}$ thick sections were cut and stained by Hematoxylene and Eosin (HE) stain. The staining for reticulin fibers with Gomori's Silver impregnation method was done in selected cases to know the extent of marrow fibrosis and grading was done. For selected cases indicating Flow cytometric analysis and cytogenetic study, marrow was collected in a heparinised tube of at least $5 \mathrm{ml}$ in volume and sent to the lab. Bone marrow biopsy and aspiration findings along with selected cases with flow cytometric and cytogenetic interpretations were analyzed in context of clinical signs, symptoms and other laboratory investigations and final diagnosis was given.

\section{Results}

Total 158 cases studied were categorized on the basis of peripheral blood pictures and clinical diagnosis was summarized in table-2[Table-2]. The results were analyzed as per the extent of correlation between BMA and BMB.

The results of the comparative evaluation was divided into 4 categories:

1. Number of cases having positive correlation between BMA and BMB

2. Diagnosis on BMB alone where BMA was not confirmatory

3. Number of cases where BMA is more confirmatory, BMBwas not contributory to the diagnosis

4. Number of cases where no opinion possible either in BMA or BMB

Table-2: Total no of cases basing on clinical diagnosis and BM evaluation

\begin{tabular}{|c|c|c|c|}
\hline - Serial no & - $\quad$ Clinical diagnosis & - $\quad$ BM diagnosis & - $\quad$ No of cases \\
\hline - 1 & - $\quad$ Fever & - $\quad$ Normal hematopoiesis & - 9 \\
\hline - 2 & - $\quad$ PUO & - $\quad$ Erythroid hyperplasia & 11 \\
\hline - 3 & - $\quad$ fever anemia & - $\quad$ Hypoplastic marrow & - 14 \\
\hline - 4 & - $\quad$ pancytopenia & - $\quad$ Aplastic anemia & - 7 \\
\hline - 5 & - $\quad$ Bleeding,peteche & • $\quad$ Thrombocytopenicpurpura & - 5 \\
\hline - 6 & - $\quad$ anemia & - $\quad$ Anemia with chr.infection & $\bullet$ \\
\hline - 7 & - $\quad$ Fever,wt loss & - $\quad$ Granuloma & - 1 \\
\hline - 8 & - $\quad$ Anemia,spenomegaly & • $\quad$ Hypersplenism & $\bullet \quad 5$ \\
\hline - 9 & $\begin{array}{l}\text { - } \quad \text { Lymphadenopathy,fever,gum } \\
\text { bleeding }\end{array}$ & - $\quad$ Acute leukemia & - 23 \\
\hline - 10 & - $\quad$ Low back pain, fracture & - $\mathrm{MM}$ & - $\quad 25$ \\
\hline - 11 & - $\quad$ Bleeding $\mathrm{P} / \mathrm{R}$ & - $\mathrm{MDS}$ & - 8 \\
\hline - 12 & - $\quad$ Anemia,splenomegaly & - $\quad$ MPN(CML) & - 11 \\
\hline - 13 & - $\quad$ Leukocytosis,lymphadenopathy & - $\quad$ CLL & - 6 \\
\hline - 14 & - $\mathrm{k} / \mathrm{c} / \mathrm{oNHL}$ & - $\quad \mathrm{NHL} / \mathrm{HL}$ & - 30 \\
\hline$\bullet \quad 15$ & - $\quad$ Presence of primary cancer & - $\quad$ Secondary metastasis & $\bullet$ \\
\hline
\end{tabular}


Among these 158 cases studied, 135 cases (85.4\%) showed there was positive correlation in BMA and BMB.Table-3 shows the spectrum of cases with positive correlation. There were 20 cases $(12.6 \%)$ where BMA was not so significant and diagnosis was possible by BMB only[Table-4]. There were 2 case(1.2\%) where BMB suggested no confirmatory result and diagnosis was based on BMA alone. Only a single case $(0.6 \%)$ was there, where BMA was inadequate and BMB showed hyper cellular or reactive marrow. In that case no opinion was possible either in BMA and BMB.

Table-3: positive correlation b/w BMA and BMB

\begin{tabular}{|l|l|l|l|}
\hline Serial no & diagnosis & No.of cases & \% \\
\hline $\mathbf{1}$ & Reactive marrow & $\mathbf{5 / 5}$ & $\mathbf{1 0 0 \%}$ \\
\hline $\mathbf{2}$ & Erythoid hyperplasia & $\mathbf{1 1 / 1 1}$ & $\mathbf{1 0 0 \%}$ \\
\hline 3 & Aplastic anemia & $\mathbf{6} / 7$ & $\mathbf{8 5 . 7 \%}$ \\
\hline $\mathbf{4}$ & ITP & $\mathbf{5 / 5}$ & $\mathbf{1 0 0 \%}$ \\
\hline $\mathbf{5}$ & Autoimmuehemolyticanemia & $\mathbf{2 / 2}$ & $\mathbf{1 0 0 \%}$ \\
\hline $\mathbf{6}$ & Act.leukemia & $\mathbf{2 2 / 2 3}$ & $\mathbf{9 5 . 6 \%}$ \\
\hline $\mathbf{7}$ & MPN(CML) & $\mathbf{9 / 1 1}$ & $\mathbf{8 1 . 8 \%}$ \\
\hline $\mathbf{8}$ & MDS & $\mathbf{7 / 8}$ & $\mathbf{8 7 . 5 \%}$ \\
\hline $\mathbf{9}$ & CLL & $\mathbf{3 / 6}$ & $\mathbf{5 0 \%}$ \\
\hline $\mathbf{1 0}$ & MM & $\mathbf{2 4 / 2 5}$ & $\mathbf{9 6 \%}$ \\
\hline 11 & NHL/HL & $25 / 30$ & $83.3 \%$ \\
\hline $\mathbf{1 2}$ & METASTASIS & $\mathbf{3 / 3}$ & $\mathbf{1 0 0 \%}$ \\
\hline $\mathbf{1 3}$ & Hypocellular marrow & $\mathbf{6 / 6}$ & $\mathbf{1 0 0 \%}$ \\
\hline $\mathbf{1 4}$ & Hypersplenism & $\mathbf{5 / 5}$ & $\mathbf{1 0 0 \%}$ \\
\hline $\mathbf{1 5}$ & Marrow with normal & $\mathbf{2 / 2}$ & $\mathbf{1 0 0 \%}$ \\
\hline Total & hematopoiessi & $135 / 158$ & $85.4 \%$ \\
\hline
\end{tabular}

Table-4: diagnosed on BMB alone

\begin{tabular}{|l|l|l|l|}
\hline Serial no & Diagnosis on BMB & No of cases & $\%$ \\
\hline 1 & CML with fibrosis & $2 / 11$ & $18 \%$ \\
\hline 2 & MDS & $1 / 8$ & $12.5 \%$ \\
\hline 3 & MM & $1 / 25$ & $0.6 \%$ \\
\hline 4 & Aplastic anemia & $1 / 7$ & $14.2 \%$ \\
\hline 5 & NHL & $5 / 30$ & $16.6 \%$ \\
\hline $\mathbf{6}$ & Granuloma & $\mathbf{1 / 1}$ & $\mathbf{1 0 0 \%}$ \\
\hline 7 & Acute keukemia & $1 / 23$ & $4.3 \%$ \\
\hline 8 & CLL & $3 / 6$ & $50 \%$ \\
\hline $\mathbf{9}$ & Myelofibrosis & $\mathbf{5 / 5}$ & $\mathbf{1 0 0 \%}$ \\
\hline Total & & 20 & \\
\hline
\end{tabular}

In our study we found highest correlation rate with the diagnosis of reactive marrow (100\%) and those cases with erythroid hyperplasia (100\%). Those cases diagnosed as erythoid hyperplasia, were further evaluated for chronic anemia and Prussian blue staining was done to estimate the iron status and along with this other biochemical parameters were taken into consideration to reach a final diagnosis. There were other cases of Hypersplenism and Autoimmune hemolytic anemia (AIHA) where 100\% correlation was obtained in both BMA and BMB study. Another 3 case of secondary metastasis to bone marrow showed $100 \%$ correlation between BMA and BMB.

Other cases with a very good positive correlation were hematological malignancies such as Multiple Myeloma, where there was $96 \%$ correlation. Out of 25 diagnosed cases, 24 cases gave a positive correlation in between BMA and BMB. Only 1 case $(0.6 \%)$ was diagnosed on BMB. Acute Leukemia showed $95.6 \%$ positive correlation and 4.4\% was diagnosed in BMB alone showing hypo cellular marrow on BMA. Out of the total 23 cases of acute leukemia, both Acute Lymphoblastic leukemia (ALL) and Acute Myeloid leukemia (AML), in 7 cases Flow cytometry analysis (FCM) was done and in 3 cases cytogenetic study was performed and their interpretations were correlated with the BMA and BMB interpretations which showed a strong link between FCM and Cytogenetic study with BMAand BMB for giving a definitive diagnosis in different hematological malignancies [Table-5 and Table-6]. Likely in Myelodysplastic Syndrome (MDS) and Myeloproliferative neoplasia (MPN) cases showed high positive correlation i.e.; $87.5 \%$ and $81.8 \%$ respectively. And here also in CML and MDS cases, cytogenetic analysis gave a strong correlation to BMA and BMB interpretations [Table6]. 30 cases were diagnosed cases of Non-Hodgkin's lymphoma (NHL), out of which 25 cases (83.3\%) showed positive correlation in BMA and BMB and another 5 cases (17.7\%) were diagnosed in BMB and BMA played inconclusive role in that case. Among these 30 cases of NHL, FCM was done in 4 cases which gave a confirmatory diagnosis [Table-5]. 
Table-5: flowcytometric interpretation along with Bone marrow findings

\begin{tabular}{|c|c|c|c|c|c|}
\hline Serialno & $\begin{array}{l}\text { Bone marrow } \\
\text { diagnosis }\end{array}$ & $\begin{array}{l}\text { No of } \\
\text { cases }\end{array}$ & FCM finding & FCM interpretations & $\begin{array}{l}\text { Final } \\
\text { diagnosis }\end{array}$ \\
\hline 1 & AML-ML & l & $\mathrm{CDl3}+, \mathrm{CD} 33+, \mathrm{HLA}-\mathrm{DR}+, \mathrm{CDllb}+$ & AML with maturation & AML-M2 \\
\hline 2 & AMK-M3 & 2 & CD13+,CD33+,CD34-,HLA-DR- & $\begin{array}{l}\text { Acute } \\
\text { leukemia }\end{array}$ promyelocytic & AML-M3 \\
\hline 3 & AML-M5 & 1 & CD13+,CD33++,CDl4+,CDllb,llc+,HLADR+ & $\begin{array}{ll}\text { Acute } & \text { monocytic } \\
\text { leukemia } & \end{array}$ & AML-M5 \\
\hline 4 & $\begin{array}{ll}\text { ALL on } \\
\text { therapy }\end{array}$ & l & TdT-,CD34- & Complete remission & $\begin{array}{l}\text { Complete } \\
\text { remission }\end{array}$ \\
\hline 5 & ALL-Ll & 2 & HLA-DR+,IdT+,CD19+,CD79at & $\begin{array}{ll}\text { Acute } & \text { lymphoblastic } \\
\text { leukemia }\end{array}$ & $\begin{array}{l}\text { ALL-B } \\
\text { lineage }\end{array}$ \\
\hline 6 & $\begin{array}{l}\text { NHL } \\
\text { (DLBCL) }\end{array}$ & 2 & $\begin{array}{l}\text { CD19+,CD20+,CD79a+,BCL-6+,CD10- } \\
\text { MUMl- }\end{array}$ & $\begin{array}{l}\text { Diffuse large B cell } \\
\text { lymphoma, NOS }\end{array}$ & DLBCL \\
\hline 7 & NHL(ALCL) & l & CD30+t+,ALK + & $\begin{array}{l}\text { ALK+re Anaplastic large } \\
\text { cell lymphoma }\end{array}$ & ALCL \\
\hline 8 & NHL(PTCL) & l & CD4+,CD10-,BCL6-,Ki 67++ & $\begin{array}{l}\text { Peripheral } \\
\text { lymphoma,NOS }\end{array}$ & PTCL \\
\hline
\end{tabular}

\begin{tabular}{|l|l|l|l|l|}
\hline Serial no & BM diagnosi & No of cases & Cytogenetic findings & $\begin{array}{l}\text { Cytogenetic } \\
\text { interpretation }\end{array}$ \\
\hline 1 & CML-CP & 3 & Bcr-Abl translocation & CML \\
\hline 2 & MDS & 1 & $\begin{array}{l}5 q-(q 31), 5 q+,-7 q,- \\
13 q,-18,+19\end{array}$ & $\begin{array}{l}\text { AML } \\
\text { myelodysplastic related } \\
\text { changes }\end{array}$ \\
\hline 3 & AML-M3 & 1 & $\mathrm{t}(15,17)$ & $\begin{array}{l}\text { Acute promyelocytic } \\
\text { leukemia }\end{array}$ \\
\hline 4 & AML-M3V & 1 & $\mathrm{t}(15,17)$ & $\begin{array}{l}\text { Acute promyelocytic } \\
\text { leukemia }\end{array}$ \\
\hline 5 & ITP & 1 & No genetic alteration & Normal karyotype \\
\hline
\end{tabular}

Out of 5 cases of ITP (immune thrombocytopenic purpura), 4 cases (80\%) showed positive correlation with the BMA and BMB reports. Only 1 case (20\%) was diagnosed basing on the BMB report where BMA gave inclusive results. 1 case of ITP was also undergone cytogenetic analysis for confirmation. In our study we got 7 cases of aplastic anemia, out of which 6 cases $(85.7 \%)$ gave positive correlation in both BMA and BMB. 1 case was inconclusive in BMA and diagnosis was made basing on BMB. In case of Mylofibrosis and CML with fibrosis of marrow were exclusively diagnosed on BMB alone and BMA had very little or no effect in these cases. Patients with lymphoproliferative disorders or chronic lymphocytic lymphoma (CLL) showed almost equal correlation $(50 \%)$ in both BMA and BMB.

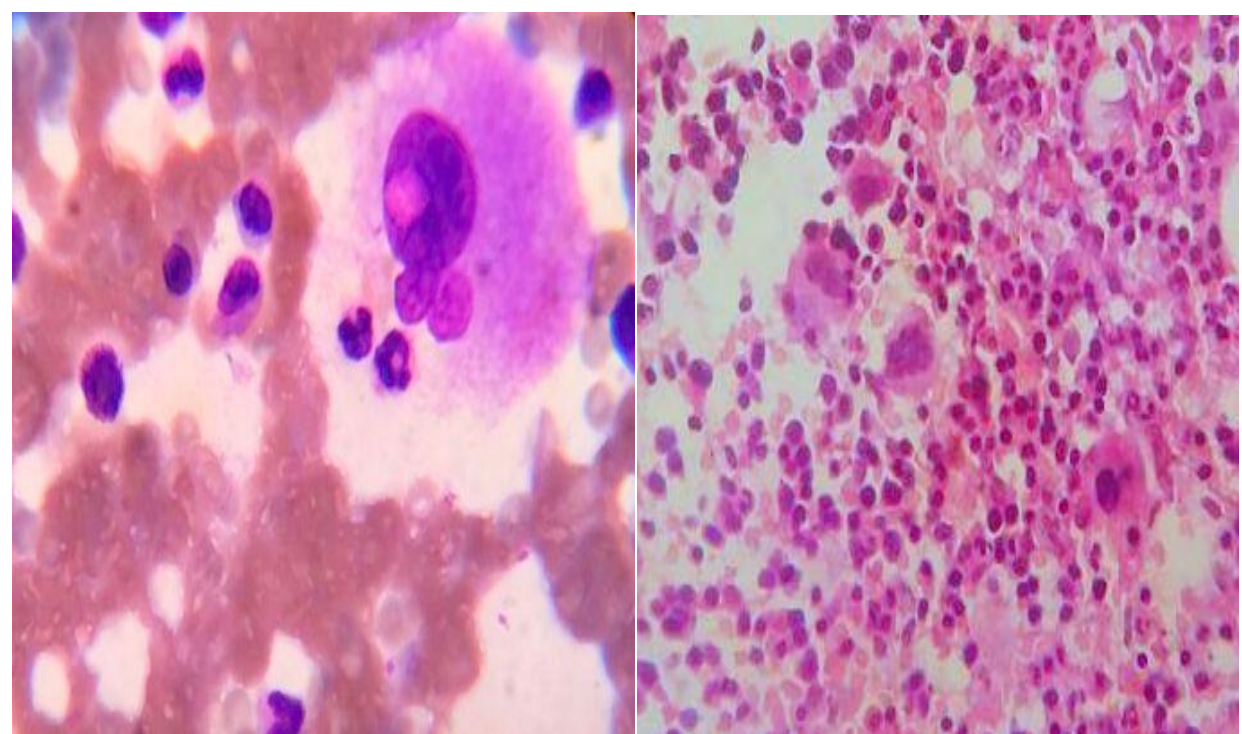

Fig 1a: BMA of ITP

Fig1b: BMB of ITP 


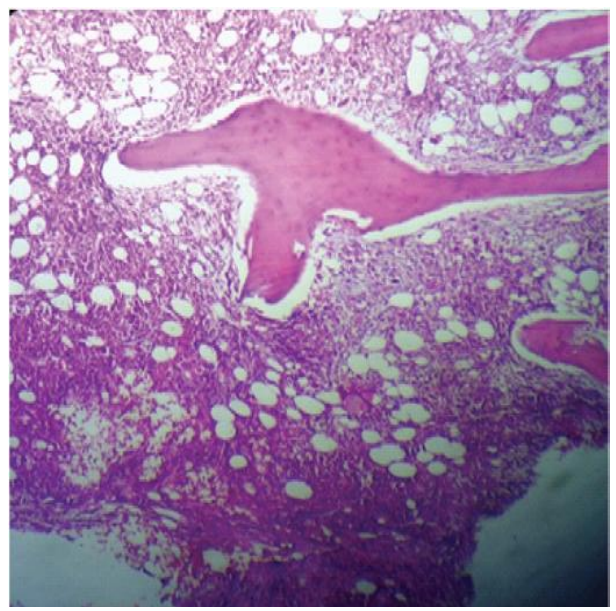

Fig2a: BMB of CML

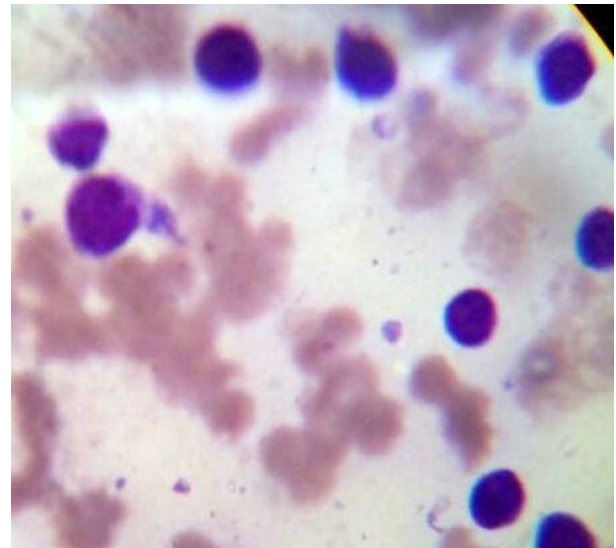

Fig3a:BMA of ALL

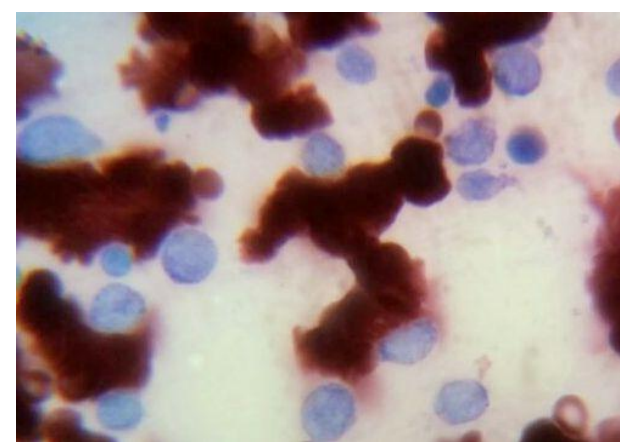

Fig 3b: SBB Staining negative

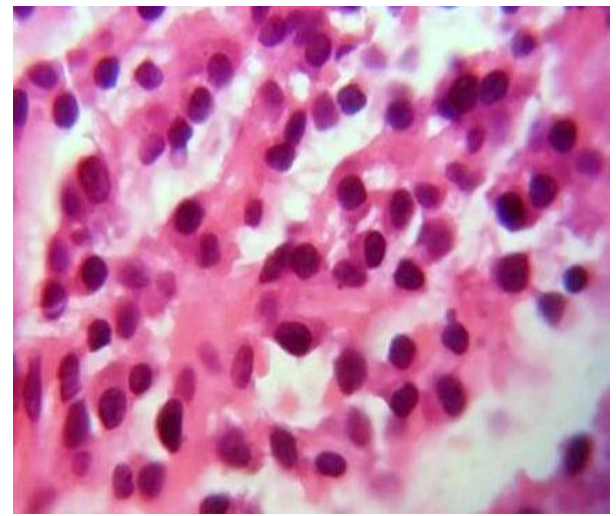

Fig 4b: BMB of MM

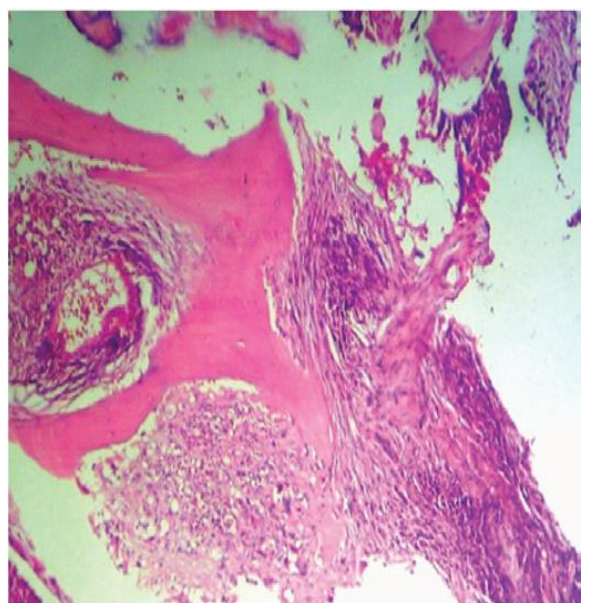

Fig2b: BMB Showing CML with fibrosis

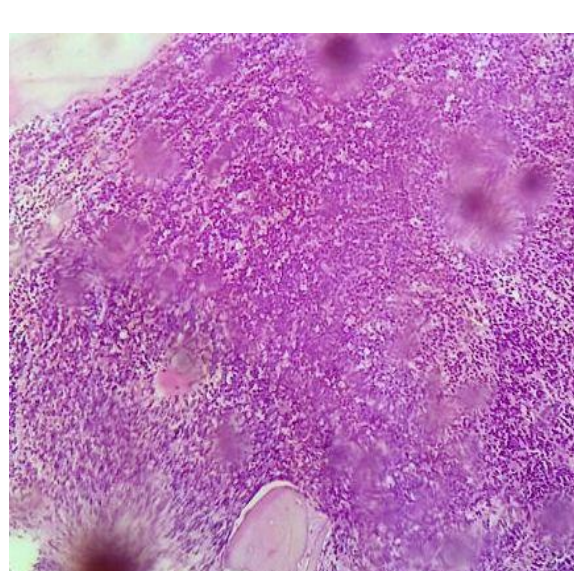

Fig 3c: BMB in ALL

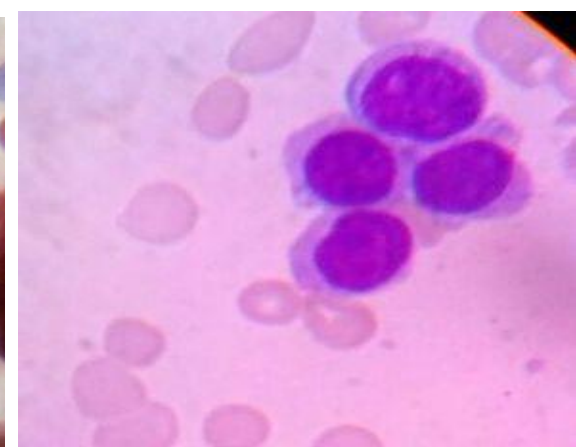

Fig 4a:BMA of MM

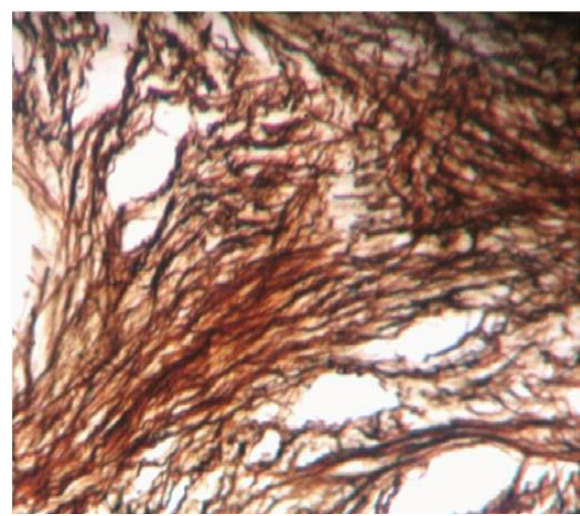

Fig 5a: Grade -3 myelofibrosis in BMB 


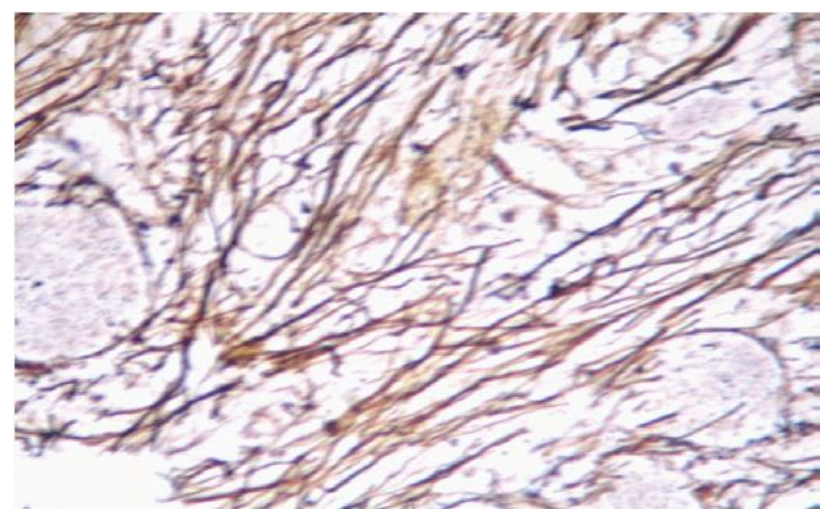

Fig5b: grade-2 fibrosis in $\mathrm{BMB}$

\section{Discussion}

The bone marrow examination is a valuable and important investigation in hematology practice. BMA and BMB are two important diagnostic tools for the diagnosis of various hematological and non-hematological disorders. These procedures are also valuable for follow-up of patients undergoing chemotherapy to know the remission status and also in those candidates undergoing for bone marrow transplantation.[21,22]. it is a well known fact that BMB and BMA are complimentary to each other. Now a days, both the procedures are simultaneously done in every indicated cases in one setting [22] and along with it samples are collected for Flow cytometry and Cytogenetic analysis to reach a conclusive diagnosis and to know the prognostic importance of the disease. In our study, we did a comparative evaluation of all BMA and BMB along with it correlating the findings of FCM and Cytogenetic to see the correlation between all these procedures, to study the advantages and disadvantages of all.

Our study comprises $85.4 \%$ positive correlation between BMA and BMB. The highest positive correlation was seen among the cases of reactive marrow and erythroid hyperplasia. These observations were nearly similar to the finding seen in a study conducted by $\boldsymbol{C h}$ Toi $\boldsymbol{P}$ et al.[7] and Manmeet Kaur et al[B]. Other cases of Hypersplenism, secondary metastasis and AIHA also showed highest positive correlations. In case of acute leukemia cases, though BMA confirms the diagnosis, bone marrow biopsy specimen complements the peripheral smear and aspirate findings in providing additional information for the diagnosis and especially the prognosis of acute leukemia [9]. With this FCM study gives accuracy to the diagnosis.

In case of Aplastic anemia, $85.7 \%$ positive correlation was found in BMA and BMB in our study.BMA suggested a hypocellular marrow where as BMB gives the qualitative and quantitative assessment of cellularity, therefore confirms the diagnosis and overcomes the limitation of BMA $[8,11]$

$96 \%$ (24/25) of Multiple myeloma showed positive correlation in BMA and BMB. In case of easy and adequate aspiration, diagnosis of $\mathrm{MM}$ can be diagnosed easily in BMA alone. In such cases BMB complemented the BMA, as it helps to identify compact masses of plasma cells with no stroma as observed by Sabrahwal et al.[22].Our study correlated with the study done by Charles et al[25] and Pampa Ch Toi et $\boldsymbol{a l}$,where they detected myeloma in trephine biopsy and simultaneous bone marrow aspirates.

We have encountered 11 cases of MPNs, out of which diagnosis was possible from Peripheral blood smear and BMA in 9 cases (81.8\%). Role of trephine biopsy is not only in differentiation of MPN but to assess the overall marrow cellularity,histo-topographic distribution of cells, morphology of megakaryocytes as well as blasts and degree of Myelofibrosis [fig5a,5b]. In our study out of 11 cases of CML , 4 cases had grade- 3 fibrosis which was established in reticulin stain of the bone marrow biopsy specimen[fig5].This highlights the importance of trephine biopsy in such cases because BMA does not have much role in diagnosis of Primary myelofibrosis.[16B]. out of these 11 cases dignosed as CML ,cytogenetic study was done in 4 cases for Bcr-Abl genetic translocation and the result was correlated with BMA and BMB reports to assess the importance of cytogenetic study in Bone marrow in recent years.

There are 6 cases of CLL, with diffuse involvement of marrow, which was seen on biopsy section while aspiration showed marrow involvement only. Out of these 6 cases, 3cases (50\%) showed positive correlation in BMA and BMB and another 3 cases $(50 \%)$ were diagnosed in BMB alone. Bone marrow examination in CLL cases should always include a trephine biopsy Because BMA gives very little information. Pattern of marrow involvement by leukemic cells could be only analyzed by trephine biopsy and BMB also gives an accurate assessment of extent of infiltration and gives prognostic importance.

But in cases of Acute leukemia though the correlation between BMA and BMB is very high, that is almost $95.6 \%$ (22/23) in our study, but the cytomorphology of neoplastic cells are more clear in BMA than in BMB. Bone marrow aspiration showed the origin of cells whether it is of myeloid or lymphoid and morphological FAB classification can be made basing upon the interpretations of aspiration. Moreover special 
staining like Sudan Black B(SBB) and Myeloperoxidase(MPO) can be done on aspiration smears to confirm the cell type. $\mathrm{BMB}$ only gives the impression on type and pattern of distribution and marrow cellularity. In the recent years in this cases flow cytometry and cytogenetic study is very important diagnostic tool to reach a definitive diagnosis. In our case out of 23 cases of acute leukemia, 13 were acute myeloid leukemia (AML) and 10 were acute lymphoblastic leukemia (ALL). Out of these 13 cases FCM was done in 4 cases ( 2 of AML-M4 and 1of AML-M5 and 1 of AML-M2) and the results were correlated with BMA and BMB interpretations [Table-5].In 2cases of AML patients diagnosed as AML-M3 and AML-M3V, cytogenetic analysis was done which was correlated with BMAandBMB. Out of 10 cases of ALL, in 2 cases FCM and 1case Cytogenetic was done and it was correlated with BMA and BMB interpretations [table 5,6]. All these studies explained there is a very strong correlation between all these procedures and though BMA is superior to BMB as morphology of the cell is concerned, but BMA, BMB, FCM and Cytogetics, all are complementary to each other to give a definitive diagnosis and to know the prognostic index of the patient.

In our study we got only a single case of granulomatous lesion in bone marrow which was diagnosed by $\mathrm{BMB}$ alone (100\%).Basing on the previous clinical history, presentation and other investigations, tuberculous granuloma was given as final diagnosis.BMB is better procedure for detecting granulomas in the bone marrow. In cases of myelodysplastic syndrome (MDS), in our study we got out of 8 cases 7 cases $(87.5 \%)$ showed positive correlation in BMA and BMB and only 1 case was diagnosed in BMB alone. Both of these procedures are complimentary to each other and the BMB in MDS gives accurate cellularity, abnormal topographic distribution of cells and also dysplastic morphology of the cells for which along with BMA, BMB procedure should be done simultaneously in such cases. We got 30 cases of non-Hodgkin's lymphoma in our study, out of which 25 cases $(83.3 \%)$ showed positive correlation and only 5 cases $(17.7 \%)$ were inconclusive in BMA and diagnosis was made on biopsy. But in all cases of NHL, the detailed biopsy study showed the marrow cellularity,topography of the cells and pattern of distribution whether diffuse, interstitial or infiltrative .So in all cases of NHL, along with BMA ,BMB should be done simultaneously both for accurate diagnosis and prognostic point of view.

Likely in case of Thrombocytopenic purpura cases, both the procedures are complimentary to each other. But an additional finding on BMB was normal arrangement of megakaryocytes which were seen in increased number. Hence from the above discussion, it was observed that bone marrow evaluation is a very effective and important diagnostic tool in hematological and nonhematological disorders. Complete evaluation of bone marrow samples includes a brief patient history, hematological profile, BMA smears, BMB materials, FCM and cytogenetic study. A correlation of BMA, BMB, FCM and Cytogenetic are complementary to each other. The bone marrow aspiration generally provides an excellent cytomorphological details which enables hematopathologists in recognizing the abnormal hematopoietic cells or non-native cells in case of nonhematological disorders. Whereas, bone marrow trephine biopsy demonstrates the topographical arrangement of hematopoietic cells within the marrow and gives more representative view of cellularity of the marrow and allows infiltration to be recognized clearly.

On the other hand flowcytometric analysis of the bone marrow sample provides definitive idea about the origin and type of the cell in different hematological malignancies and adds a strong diagnostic confirmation on the BMA and BMB interpretations. Simultaneous cytomorphological study now days put an important impression on the hematological disorders, providing a definitive evidence of genetic alteration in different hematological malignancies for the purpose of knowing the prognostic index, patient's survival and targeted therapy.

\section{Conclusion}

The present study showed that BMA and BMB are easy, rapid, cost-effective and more or less are of equal value in various hematological and non-hematological disorders of bone marrow. Although both are complementary to each other on correlation, but one or other of these methods is more conclusive. In our experience, we felt that for diagnostic purpose both the procedures can be done simultaneously as BMA gives better morphology of the cells whereas BMB provides more accurate information regarding the distribution of cells within the marrow, grading of fibrosis, pattern of infiltration in lymphomas and granulomas. BMB especially useful in cases of tuberculosis, myelodysplastic syndromes, and myeloproliferative disorders and non-Hodgkin's lymphomas. We also felt that corroborative study of flowcytometry and cytogenetic, particularly in cases of hematological malignancies gives confirmatory evidences to give a definitive diagnosis . Though both of these procedures are little costly, still to know the definitive genetic alteration ,origin of the neoplastic cells and the remission status after therapy, in every cases of hematological malignancies along with routine bone marrow aspiration and biopsy ,flow cytometry and cytogenetic study should be included in the investigating regimen. 


\section{References}

[1]. Neal S, Young. Aplastic anaemia, myelodysplasia and related bone marrow syndromes. In: Kasper, Braunwald, Fauci, Hauser, Longo, Jameson, editors. Harrison's Principles of Internal medicine(Vol. 1).16. New Delhi: McGraw Hill;2005.p.617.

[2]. Krause JR. An appraisal of the value of the bone marrow biopsy in the assessment of proliferative lesions of the bone marrow. Histopathology. 1983;7(5):627-44.

[3]. Gale E, Torrance J, Bothwell T. The quantitative estimation of total iron stores in human bone marrow. $\mathrm{J}$ Clin invest. 1963;42(7):1076-82.

[4]. Bauermeister DE. Quantitation of bone marrow reticulin- a normal range. Am J Clin Pathol. 1971;56:24-31.

[5]. Riley RS, Hogan TF, Pavot DR, Forysthe R, Massey D, Smith E, et al. A pathologist's perspective on bone marrow aspiration and biopsy; Performing a bone marrow examination. J Clin Lab Anal. 2004;18(2):70-9.

[6]. Islam A. Bone marrow aspiration prior to bone marrow core biopsy using the same bone marrow biopsy needle. A good or bad practice. J Clin Pathol. 2007;60:212-15.

[7]. Ch Toi P, Varghese G'Boy R, Rai R. Comparative evaluation of simultaneous bone marrow aspiration and bone marrow biopsy. An institutional experience. Indian J Hematol Blood Transfus. 2010;26(2):41-44.

[8]. Stuart_Smith SE, Hughes DA and Bain BJ. Are routine iron stains on bone marrow trephine biopsy specimens necessary? J Clin Pathol. 2005;58(3):269-72.

[9]. Younus U, Saba K, Aijaz J, Bukhari MH, Naeem S. Significance of Bone Marrow Histology in the Diagnosis of Acute Myeloid Leukemia. ANNALS. 2011;17(1):5-8.

[10]. Krober SM, Greschniok A. Acute lymphoblastic leukemia: correlation between morphological/immunohistochemical and molecular biological findings in bone marrow biopsy specimens. Mol pathol. 2000; 53(2):83-87.

[11]. Milosevic R, Jankovic G, Antonijevic N, Jovanovic V, Babic D, Colovic M. Histopathologic characteristics of bone marrow in patients with aplastic anaemia. Srp Arh Celok Lek. 2000; 128(5-6): 200-04.

[12]. Bain BJ. Clark DM, Wilkins BS, editors. Bone marrow pathology. 4th ed. Oxford, UK: Wiley Blackwell Publishing; 2010. pp. 11.

[13]. Humphries JE. Dry tap bone marrow aspiration: clinical significance. Am J Hematol. 1990;35(4):247-50.

[14]. Babarovic E, Valkovic T, Stifter S, Budisavljevic I, Seili-Bekafigo I, Duletic-Nacinovic A, et al. Assessment of Bone Marrow Fibrosis and Angiogenesis in Monitoring Patients With Multiple Myeloma. Am J Clin Pathol. 2012;137:870-78.

[15]. Yookarin KH, Basu D, Dutta DK. Bone marrow trephine biopsy findings in chronic myeloid leukemia. Malaysian Journal of pathology. 2002;24:37-43

[16]. Mahajan V, Kaushal V, Thakur S, Kaushik R. A comparative study of bone marrow aspiration and bone marrow biopsy in haematological and non haematological disorders -An institutional experience. JIACM. 2013;14(2):133-35.

[17]. Goyal S, Singh UR, Rusia U. Comparative Evaluation of Bone Marrow Aspirate with Trephine Biopsy in Hematological Disorders and Determination of Optimum Trephine Length in Lymphoma Infiltration. Mediterr J Hematol Infect Dis. 2014;6(1):e2014002. doi: 10.4084/MJHID.2014.002. eCollection 2014

[18]. Kim YS, Ford RJ, Faber JA. B-cell chronic lymphocytic leukemia/small lymphocytic lymphoma involving bone marrow with an interfolicular pattern. Am J Clin Pathol. 2000;114(1):41-46.

[19]. Steven J, Jubelirer. The role of the bone marrow examination in the diagnosis of immune thrombocytopenic purpura: case Series and Literature Review. Clinical and Applied Thrombosis/Hemostasis. 2002;8:173-76.

[20]. B J Bain. Bone marrow aspiration. J Clin Pathol. 2001; 54:657-63.

[21]. Riley RS, Hogan TF, Pavot DR, Forysthe R, Massey D, Smith E,Wright L Jr, Ben-Ezra JM (2004) A pathologist's perspective on

[22]. bone marrow aspiration and biopsy; Performing a bone marrowexamination. J Clin Lab Anal 18:70-79

[23]. Islam A (2007) Bone marrow aspiration prior to bone marrow core biopsy using the same bone marrow biopsy needle. A good

[24]. or bad practice. J Clin Pathol 60:212-215Sabharwal BD, Malhotra V, Aruna S, Grewal R (1990) Comparative evaluation of bone marrow aspirate particle smear, imprints and biopsy sections. J Postgrad Med 36:194-198

[25]. Bain BJ, Clark DM, Lampert IA, Wilkins BS (eds) (2001) Bone marrow pathology, 3rd edn. Blackwell Science, Italy, pp 372-373

[26]. Charles KS, Winfield DA, Angel C, Goepel J (2004) Audit of bone marrow aspirates and trephine biopsies in multiple myelomaa single centre study. Clin Lab Hematol 26:403-406

[27]. Cotelingam JD (2003) Bone marrow biopsy: Interpretive guidelines for surgical pathologists. Adv Anat Pathol 10:8-26

[28]. Moid F, Depalma L (2005) Comparison of relative value of bone marrow aspirates and bone marrow trephine biopsies in the

[29]. diagnosis of solid tumour metastasis and Hodgkin Lymphoma. Arch Pathol Lab Med 129:497-501

[30]. Pasquale D, Chikkapa G (1986) Comparative evaluation of bone marrow aspirate particle smears, biopsy imprints and biopsy [31]. sections. Am J Hematol 22:381-389

[32]. Barekman CL, Fair KP, Cotelingam JD (1997) Comparative Utility of diagnostic bone marrow components: a 10 year study.

[33]. Am J Hematol 56:37-41

[34]. Atac B, Lawrence C, Goldberg SN (1991) Metastatic tumor: the complementary role of the marrow aspirate and biopsy. Am J Med Sci 302:211-213

[35]. Ozkalemkas F, Ali R, Ozkocaman V, Oscelik T, Ozanu OH et al (2005) The bone marrow aspirate and biopsy in the diagnosis of unsuspected non hematologic malignancy. A clinical study of 19 cases. BMC Cancer 1(5):1443

[36]. Howell SJ, Grey M, Chang J (2002) The value of bone marrow examination in the staging of Hodgkin Lymphoma. A review of

[37]. 955 cases seen in regional cancer centre. Br J Haematol 119:408-411

[38]. Sharma S, Ahuja A, Murari M (2004) Bone marrow biopsy in Hodgkin's disease. Indian J Pathol Microbiol 47:364-366

[39]. [34]. Bhargava V, Farhi DC (1988) Bone marrow granulomas, clinicopathological findings in 72 cases and review of the literature.

[40]. Hematol Pathol 2:43-50

[41]. Indian J Hematol Blood Transfus (Apr-June 2010) 26(2):41-44

* Raka Hota. "A Comparative Evaluation of Simultaneous Bone Marrow Aspiration And Bone Marrow Biopsy Interpretations In Routine Hematology Practice with Special Reference to Flow Cytometry And Cytogenetic Analysis ." IOSR Journal of Dental and Medical Sciences (IOSRJDMS) 16.7 (2017): 53-60. 